\title{
Focus variation technology as a tool for tissue surface characterization
}

\author{
Jürgen Reitbauer $\cdot$ Franz Harrer $\cdot$ Rene Eckhart $[$ Dolfgang Bauer
}

Received: 29 January 2021 / Accepted: 15 May 2021 / Published online: 28 May 2021

(C) The Author(s) 2021

\begin{abstract}
The surface of tissue paper is relatively complex compared to other paper grades and consists of several overlapping structures like protruding fibres, crepe and fabric-based patterns at different spatial frequencies. The knowledge of tissue surface characteristics is crucial when it comes to improvement with respect to surface softness and the perceptual handfeel of tissue products. In this work we used the optical based, non-contact measurement principle of focus variation for surface characterization of drycreped, textured and through air dried (TAD) tissue. Based on the three tissue grades, a procedure which includes the characterization of the whole tissue surface throughout different scales within one setup, was developed. Surprisingly, focus variation was rarely used in tissue-related research, as it provides robust and reliable 3D surface information which can be used for further areal surface analysis. Special attention was given to the preparation and discussion of the raw data up to the final analysis including
\end{abstract}

several spatial filtering steps. Enhanced surface parameters like the developed interfacial area ratio (Sdr) and the power spectral density (PSD) were used to describe the surface adequately. The surface roughness of the three tissue grades was compared, with the textured tissue showing the highest roughness in Sdr and PSD analysis. Although both methods are based on different principles, a high correlation in terms of evaluated roughness is evident. Regular structures like crepe and patterns are obtainable as peaks at the respective frequency with a certain intensity in the PSD evaluation. Apart from topography in terms of structures and roughness, the wide field of view of the focus variation measurement also allows assessment of effects related to flocculation and sheet formation. The developed procedure could also be appropriate for other fibre based materials and/or fabrics, which are similar to tissue with respect to optical properties such as for example nonwovens.

J. Reitbauer · R. Eckhart $(\bowtie) \cdot$ W. Bauer Institute of Bioproducts and Paper Technology, Graz University of Technology, Inffeldgasse 23, 8010 Graz, Austria

e-mail: rene.eckhart@tugraz.com

F. Harrer

Andritz AG, Stattegger Strasse 18, 8045 Graz, Austria 


\section{Graphic abstract}

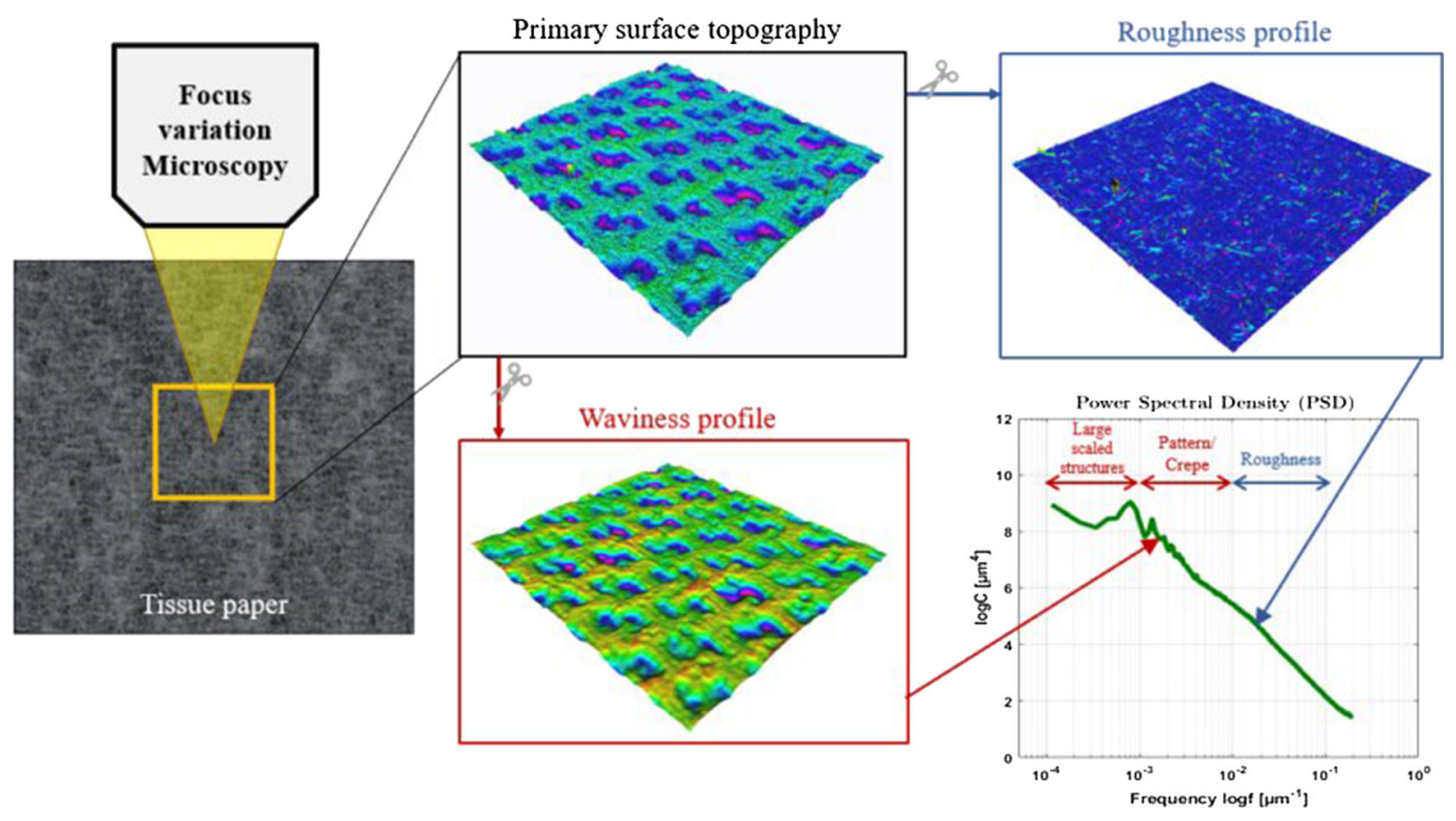

Keywords Focus variation microscopy - Tissue Surface - Surface roughness - Power spectral density · 3D Imaging

\section{Introduction}

In contrast to other paper grades, tissue paper is often in intensive contact with the human skin, especially facial and toilet tissue. Besides the functionality, the subjective perception of the overall quality and the softness of the tissue plays a major role when it comes to a purchase decision of the costumer. de Assis et al. (2018) carried out comprehensive work on the importance of softness and classified it with other tissue properties like strength and absorbency. Tissue manufacturers are well aware of this fact and constantly strive to improve tissue quality regarding the softness and handfeel properties. Softness, however, depends on many physical properties which can be related either to a surface or bulk component (Hollmark 1983). When it comes to bulk softness, paper stiffness, respectively paper flexibility plays a major role and is assessed in various ways (Hollmark and Ampulski 2004; Ko et al. 2017; Park et al. 2019).
The other major component is tissue surface softness which is consequently depending on the structural surface characteristics. As already mentioned, the perception "soft" differs between each person, nevertheless panel tests are an accepted method to validate tissue samples regarding their softness and were frequently studied to evaluate various surface softness methods (Furman et al. 2010; Rosen et al. 2014). Due to the complexity of panel tests, instrumental measurements like the Emtec tissue softness analyser (TSA) would be preferable, yet a correlation to the panel tests is not always given (Wang et al. 2019). Independent from the analytical tool of softness evaluation, the surface structure and topography of the tissue affects the results.

The measurement techniques for tissue surface characterization can be classified in contact and noncontact methods. An early approach of tissue surface characterization based on a contact method was done by Kawabata (1980) and Rust et al. (1994) with mechanical stylus scanning. The principle of a mechanical stylus instrument is based on a line scan of the surface. Based on the obtained height profile further surface related parameters can be evaluated, nonetheless the measurement strongly depends on the type of the used stylus (Ko et al. 2019). Nowadays, 
especially the stylus based surface roughness and friction measurement device KES (Kawabata Evaluation System) from Kato-Tech is used frequently for surface roughness and softness characterization (Lee et al. 2017; Ko et al. 2019; de Assis et al. 2019). The main drawbacks of the contact surface evaluation are the direct impact of the stylus on the tissue surface and the only line based information of the height data.

By the use of non-contact methods the drawbacks of the stylus based methods can be avoided however, due to the optical measurement principle other problems occur, which are summarized and discussed for different devices elsewhere (Leach 2011). A comparison of different optical methods for the analysis of surface roughness on various papers grades was done by Mettänen and Hirn (2015). One big advantage of optical methods is the areal representation of the surface. Raunio et al. (2018) used the method of photometric stereo to characterize the $3 \mathrm{D}$ structure of belt creped tissue paper based on greyscale images. In another study from Raunio et al. (2012) an optical reflectance method was used to characterize the influences of basis weight variations on the crepe frequency. Rosen et al. (2014) studied topographic modelling of haptic properties of tissue with surface parameters gained by digital light projection. A different approach was used by Gigac (2019) who used photoclinometry (inclined illumination), to relate surface softness with optical surface variability. Confocal laser scanning microscopy (CLSM) is an often used and accurate method to characterize the 3D topography of the tissue surface. Furman et al. (2010) used the CLSM for surface characterization of different tissue types, and correlated one roughness parameter successfully with softness from a panel test. A detailed study of the crepe structure and its dependence on the crepe blade lifetime for dry-creped tissue was performed by Ismail et al. (2020) using a CLSM. They further linked the topographical data with surface softness and handfeel properties, nevertheless they did not use any areal surface parameter for evaluation.

Areal surface analysis is necessary for tissue, since compared to other paper products, tissue has a complex surface containing crepe and pattern structures with a low network density and protruding fibres. These factors strongly influence some common roughness parameters like Sq (root-mean-square based surface height) and $\mathrm{Sa}$ (arithmetic mean based surface height). Therefore an interpretation of these parameters is often not reasonable for very rough surfaces like tissue. An overview of the prevalent surface parameters and their underlying concepts is given by Leach (2013). The developed interfacial area ratio (Sdr) is an areal based surface parameter and describes the surface roughness of tissue satisfactorily. Furman et al. (2010) already used this parameter in their CLSM measurements successfully for the surface roughness evaluation of tissue paper. Still, this surface parameter was rarely used in related research. Another method to characterize the tissue surface is the power spectral density (PSD) discussed in detail by Jacobs.et al. (2017). Pawlak and Elhammoumi (2011) applied PSD analysis in tissue related research to evaluate the surface softness based on line data of a lateral cut.

The chosen optical method used in this work is an infinite focus measurement (IFM) device. The measurement is based on optical focus variation only, whereas CLSM uses an additional laser for topography evaluation, hence the investment costs for a CLSM are generally higher. The IFM is a robust and accurate optical method with high resolution. An overview of the technique and its application in various fields is presented by Danzl et al. (2011). In the field of paper industry the technology was rarely used. Lechthaler and Bauer (2006) compared standard paper roughness measurements for graphical papers with the surface roughness measured with the IFM device and correlated several surface parameters. The influence of different magnifications on the results of surface parameters of office paper using the focus variation technique and a multi-scale roughness evaluation was observed by Vernhes et al. (2008). One tissue paper related work was presented by Wanske et al. (2008) using the IFM for the investigation of surface roughness changes due to ultrasonic penetration however, only two height based surface parameters were used for characterization.

In this work the measurement with the IFM was performed at three different magnifications to determine structures like crepe, fabric based patterns and roughness of dry-creped, textured and TAD tissues. The measurement and subsequent preparation and processing steps like filtering of the $3 \mathrm{D}$ datasets are described in detail. Enhanced areal surface evaluation was done using Sdr and PSD as parameters. The high field of view of the IFM allows an additional 
assessment of formation attributed effects which is also briefly discussed. With the obtained knowledge of the surface characteristics at all frequencies, effects on the surface due to the production process can be recognized and optimized. The developed procedure and the resulting parameters enable a comprehensive approach regarding the handfeel respectively surface softness properties in future work. With the flexibility of areal surface analysis and the advantages of the IFM, regions of interests throughout the tissue surface can be investigated in detail, while the temporal effort is low. This work provides an insight into the use of focus variation technology for tissue surface characterization in a novel and effective way.

\section{Materials and methods}

\section{Materials}

For surface topography evaluation three different tissue grades were used. The values for the basis weight (EN ISO 12625-6) and caliper (EN ISO 12625-3) are given in Table 1. All three tissue grades consist of a blend of softwood and hardwood kraft fibres. The production however, is based on different manufacturing technologies. The most common type is the dry-creped toilet tissue, where the surface structure is mostly determined by the dry-creping process at the Yankee cylinder. The TAD surface structure depends on the machine configuration but mainly on the structure of the applied TAD fabric. Textured tissue is based on a hybrid technology of $\mathrm{TAD}$ and dry-crepe machine concepts. The surface structure is, like TAD, predetermined by the type of fabric, nonetheless the dewatering processes are not comparable. These differences in dewatering technologies yields different properties regarding bulk and surface structure. The surface analysis was always

Table 1 Overview of the tissue samples

\begin{tabular}{llll}
\hline Parameter & Dry-creped & Textured & TAD \\
\hline Basis weight $[\mathrm{gsm}]$ & 17.2 & 19.9 & 19.5 \\
Caliper $[\mu \mathrm{m}]$ & 110 & 288 & 333 \\
Grade & Toilet & Bath & Bath \\
\hline
\end{tabular}

performed on the Yankee side of the 1-ply tissue samples.

Infinite focus measurement device

For the surface topography measurements an infinite focus measurement (IFM) device from Bruker-Alicona (Graz, Austria, Model G3) was used. The mode of operation of the IFM is based on detecting the best focus position of an optical element towards the tissue sample, which is related to a certain distance (Danzl et al. 2011). By repeating the procedure for many lateral positions, a 3D height map of the sample is generated. Besides the obtained 3D data the optical depth field color image of the surface is also acquired. The color image can subsequently be used to determine the optical formation based on Fourier analysis. An overview of the used magnifications and the respective resolutions is given in Table 2. The same area is observed with three different magnifications $(5 x, 10 x, 20 x)$ to determine the various size-based structural patterns like roughness, crepe, fabric-based patterns and formation. For each magnification the vertical resolution depends on the size of the relevant structures (crepe, fabric, single fibres). The lateral dataset size takes into account the size of given structures, hence a certain periodicity is necessary for an accurate analysis.

Areal surface analysis

Preprocessing The areal surface analysis of the measured 3D datasets was carried out with the MeasureSuite 5.3.5. software from Bruker-Alicona. All of the necessary surface topography features like roughness, crepe and structures were extracted using this software according to a defined procedure, including three preparation steps (see Figure 1). First, the main errors and artefacts due to optical interferences and tissue irregularities (e.g. holes) in the measurement data have to be corrected. Measurement artefacts are visible as isolated steep 3D peaks and have a tremendous influence on areal surface analysis, hence they were removed with the application of a maximum flank-angle condition. Areal surface analysis depends on a continuous 3D dataset, therefore missing data points due to holes in the tissue have to be filled by interpolation between the nearest adjacent 
Table 2 Overview of the used magnification and their corresponding resolution and error data

\begin{tabular}{lllll}
\hline Magnification & Vertical resolution & Lateral dataset size & Lateral pixel size & Lateral error \\
\hline $5 \times$ & $2 \mu \mathrm{m}$ & $15 \times 15 \mathrm{~mm}$ & $3.195 \mu \mathrm{m}$ & $2.62 \mathrm{~nm}$ \\
$10 \times$ & $1 \mu \mathrm{m}$ & $8 \times 8 \mathrm{~mm}$ & $1.597 \mu \mathrm{m}$ & $2.67 \mathrm{~nm}$ \\
$20 \times$ & $800 \mathrm{~nm}$ & $3 \times 3 \mathrm{~mm}$ & $795.3 \mathrm{~nm}$ & $1.35 \mathrm{~nm}$ \\
\hline
\end{tabular}

\section{Dataset with holes and artifacts}

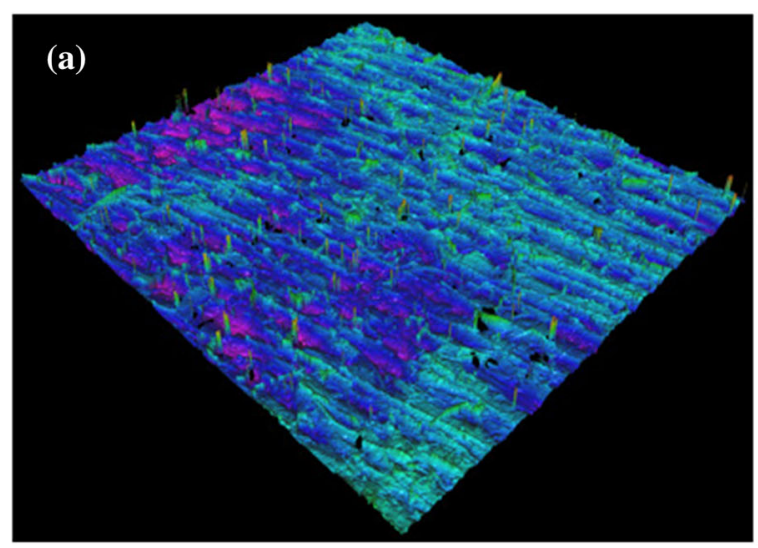

Filled holes and smoothed artifacts

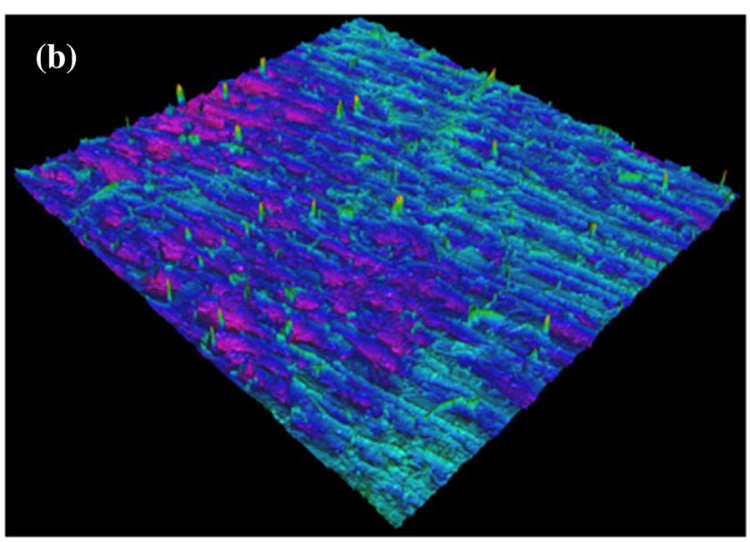

Fig. 1 a Raw 3D dataset of the infinite focus measurement device containing errors like optical artefacts and missing data points due to the holes in the tissue network. $\mathbf{b}$ Corrected dataset with filled holes and smoothed artefacts. c Removal of large

data points. It has to be noticed that such irregularities, despite the correction, always have a minor influence on the results of the areal surface analysis and cannot be completely avoided. The second step contains the elimination of the nominal form (e.g. in-plane deviations) with the use of a so called F-filter (Form-filter). During the measurement the tissue sheet is fixed with

\section{Removed in-plane deviations}

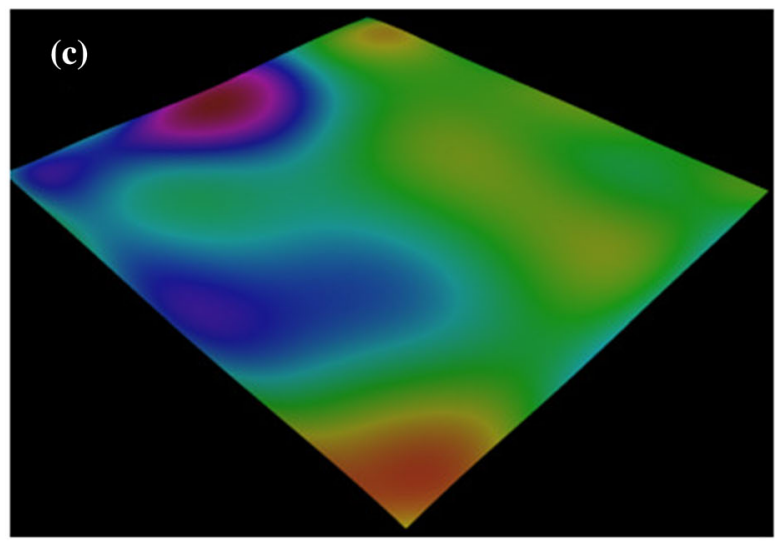

\section{Prepared dataset for filtering}

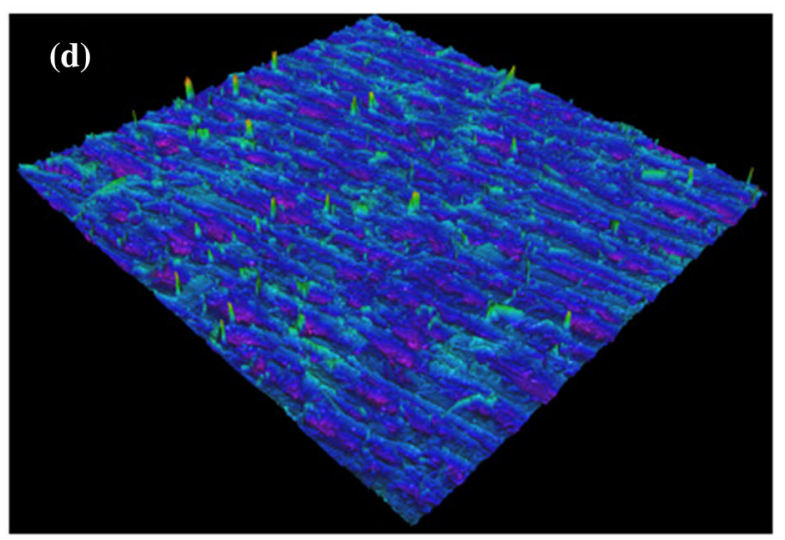

scale lateral plane deviations occurring during measurement d Dataset after preprocessing for further filtering including the automatically fitted reference plain (invisible)

an adhesive tape to a black background paper (for optical reasons), thus small planar variances occur. The last preparation step includes the definition of a spatial reference plane, which is the base for all subsequent calculations.

Filtering Following the preprocessing of the 3D dataset, further filtering techniques are applied to 
obtain the required topographical information. Filtering is important for a proper surface characterization, since several common surface parameters like $\mathrm{Sa}, \mathrm{Sz}$ and also the used Sdr could be erroneous due to superimposition of structural features. The effect of filtering on the Sdr is discussed in a further section in detail. The procedure from the preprocessed data to the final parameters is illustrated in Fig. 2. Based on the preprocessed primary data $(\mathrm{P})$, the roughness $(\mathrm{R})$ and waviness $(\mathrm{W})$ profile is separated with a cut-off wavelength of $80 \mu \mathrm{m}$, which is called nesting-index by Blateyron (2006). The applied nesting index of $80 \mu \mathrm{m}$ is valid for all used tissue grades at all magnifications and is determined with an empirical approach specifically for the used dataset and measurement configuration. For the filtering process itself a second order robust Gaussian filter for arbitrary planes (ISO 16610-71) was used. The filter considers the edge effects of inclined, as well as curved planes and has a low sensitivity towards defects like outliers and holes. However, the computational effort is high.
Developed interfacial area ratio (Sdr) The developed interfacial area ratio (Sdr) is used as one out of two parameters to describe small scale structures (i.e. roughness). The principle of the Sdr (ISO 25178) is shown in Fig. 3 and is used as a measure of the surface complexity i.e. roughness.

$S d r=\frac{A_{\text {developed }}-A_{\text {projected }}}{A_{\text {projected }}} * 100$

The Sdr describes the difference between the developed (true) and the projected surface area in percent (see Eq. 1), with a totally flat and smooth surface having a $\mathrm{Sdr}=0$

Power spectral density (PSD) In a comprehensive study Jacobs.et al. (2017) defined the power spectral density (PSD) as a tool to obtain the different spatial frequencies of a surface by Fourier transformation of the autocorrelation function signal. In surface topography analysis the PSD provides information on the appearance of periodic structures, like crepe and fabric

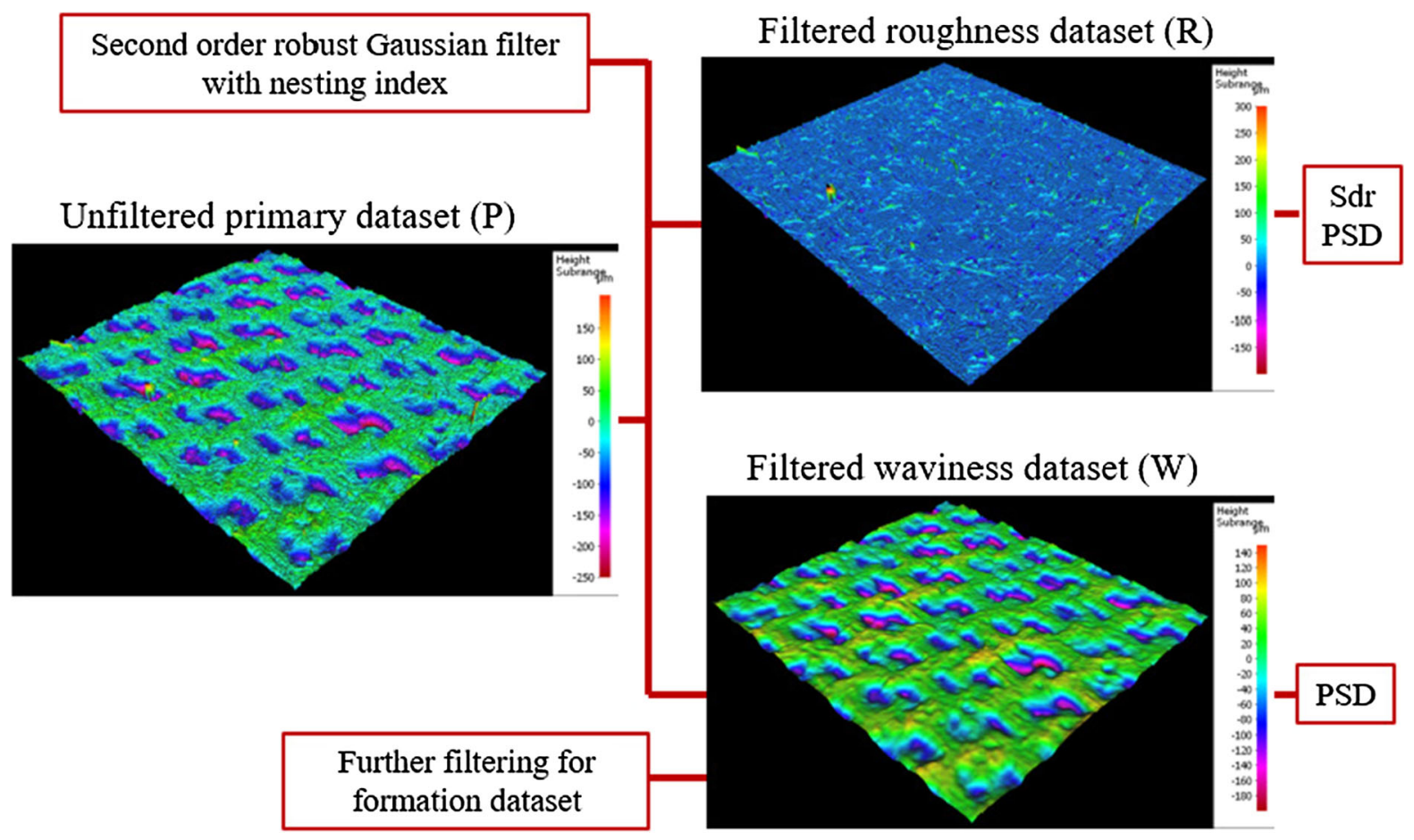

Fig. 2 The preprocessed unfiltered primary dataset $(\mathrm{P})$ is divided into a roughness and a waviness dataset by use of a second order robust Gaussian filter. The nesting index (cut-off wavelength) is $80 \mu \mathrm{m}$. The roughness dataset (R) contains information regarding the small scaled surface structures (smaller than the nesting index), while the waviness dataset
(W) consists of the large scale structures. From both datasets, the PSD curve is evaluated, whereas the Sdr value is reasonable for the roughness dataset only. For the topographical variance analysis the waviness dataset (W) is further filtered to eliminate smaller structures (e.g. crepe) 
(a) $\mathrm{Sdr}=\mathbf{2 2 0} \%$

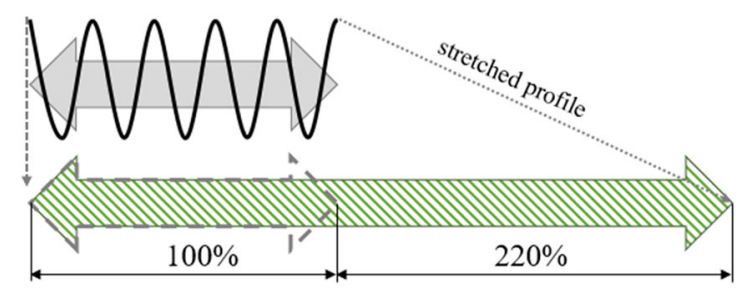

(b) $\mathrm{Sdr}=\mathbf{3 0} \%$

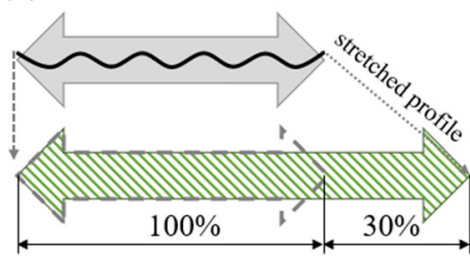

Fig. 3 The developed interfacial area ratio (Sdr) is used as a parameter for roughness evaluation. It describes the difference between the developed (true) and the projected surface area in percent, which is shown exemplary. The higher the $\operatorname{Sdr}$ (a), the higher is the complexity and hence the roughness of the surface. (Redrawn from Bruker-Alicona (2019))

patterns, which can be related to a certain spatial frequency range respectively structural size. These periodic structures are obtainable as peaks in the PSD curve. The surface roughness can also be determined and is defined by the height of the curve within the roughness frequency range. The peaks are summarized over the frequency range and are depicted as a distribution over all frequencies. When comparing the roughness of various tissue samples, differences in the height of the PSD curves occur. The higher the level of the PSD curve, the higher is the surface roughness and vice versa. For a $1 \mathrm{D}$ line profile the concept is explained schematically in Fig. 4.

In case of our work, the analysed surface data contains 3D structures, therefore the logarithmic power spectral density $(\log C)$ on $y$-axis changes to a power of four $\left(\mu \mathrm{m}^{4}\right)$ while the logarithmic spatial frequency on $\mathrm{x}$-axis remains as $\mu m^{-1}$. For simplicity reasons the surface is represented by a 1D PSD curve as it is shown in Fig. 4d. Due to the simplification and the anisotropic features of tissue a separate evaluation in machine direction (MD) and cross direction (CD) is necessary for the examination of structural properties like e.g. crepe or fabric patterns.

For roughness related properties a combined and averaged PSD curve is suitable, as roughness features (a)

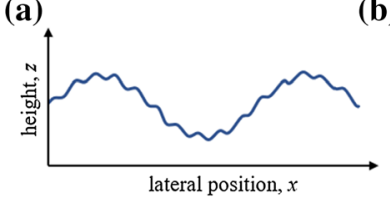

(c)

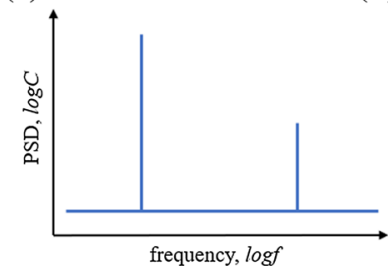

(d)
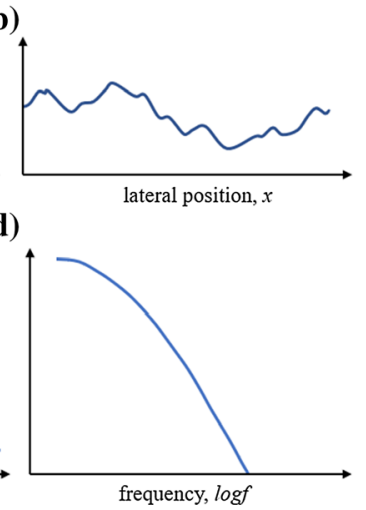

Fig. 4 The power spectral density (PSD) curve is shown for two different 1D profiles, with the quadratic mean height being the same. Profile a consists of two overlapping sinus waves, while profile $\mathbf{b}$ contains several frequencies. The PSD curve $\mathbf{c}$ shows the two sinus waves from profile a as peaks (power spectral density $\log$ C) at different frequencies (logf). The PSD curve of an arbitrary profile as for $\mathbf{b}$ is shown in $\mathbf{d}$. (Redrawn from Jacobs.et al. (2017))

occur in all directions randomly. The maximum structural size that can be assessed depends on the field of view (see lateral dataset size in Table 2). The minimum structural size that can be reasonably assessed depends on the lateral pixel size, which is also listed in Table 2 for the specific magnifications.

Optical variance analysis

The field of view of the infinite focus device and the ability to acquire optical images simultaneously with the topography measurement allows optical variance analysis using a Fast-Fourier Transformation (FFT). The optical image from the lowest magnification $(5 \mathrm{x})$ is suitable to obtain optical variances at a scale of 2-5 $\mathrm{mm}$. Image normalization and variance analysis based on the FFT algorithm were carried out in MATLAB (MathWorks, Massachusetts). The results are shown displayed as a variance distribution, where the unitless logarithmic variance is plotted over the logarithmic wavelength $\lambda(\mathrm{mm})$.

\section{Results and discussion}

PSD evaluation

As already discussed (see Figure 2) roughness (R) and waviness (W) datasets were separated with the nesting 
index of $80 \mu \mathrm{m}$ from the primary $(\mathrm{P})$ dataset. By the use of this filtering technique, typical patterns and structures like e.g. crepe can be determined and described, as presented by Rosen et al. (2014). On the other hand the surface roughness is accessible for analysis without the underlying topographical structure that might affect the used roughness parameters. This allows for example the evaluation of the effect of the morphological characteristics of different fibre types on the surface roughness. While the PSD, as it is applied in this work, does not provide any numerical values like e.g. $\mathrm{Sa}, \mathrm{Sz}$ or $\mathrm{Sdr}$ it does allow evaluation of the complete structural size spectrum of the given surface within one curve. Figure 5 shows the spatial averaged PSD curves for a dry-creped tissue surface, which was acquired at a magnification of 10x. In this figure the - in terms of direction - averaged PSD data is used for simplicity reasons. Still, when assessing structural properties, a detailed discussion in terms of $\mathrm{MD}$ and $\mathrm{CD}$ is necessary as it is shown below when comparing tissue grades. The diagram shows the PSD curve from the primary and also the incidental roughness and waviness data and gives an overview of the affected frequency ranges. The primary curve (red) includes all frequencies and has an overlap with the waviness curve (green) at lower frequencies (inverted x-axis), while the roughness curve (blue) has an overlap with the primary curve at higher frequencies.

As described in the "Methods" section, the surface roughness is characterized by the height of the PSD curve in the roughness range. Periodic structures like crepe or patterns from the fabric can be obtained as peaks in the curve. Such a dominant peak is, for example, visible in the averaged primary and waviness curve at a frequency of $4 * 10^{-3} \mu \mathrm{m}^{-1}$ which equals a structural size of $250 \mu \mathrm{m}$ (40 crepes $/ \mathrm{cm})$. The crepe appears very regularly at this structure size. Deviations in the creping process, as observed by Ismail et al. (2020) due to blade wear, could be determined as peak shifts towards a higher or smaller frequency or to a less dominant and wider, or even to several small peaks. To detect possible effects attributed to formation in the PSD, the chosen magnification is only partly suitable as the lateral size is too small. This matter is discussed in the following section. In summary, the primary dataset contains the surface information

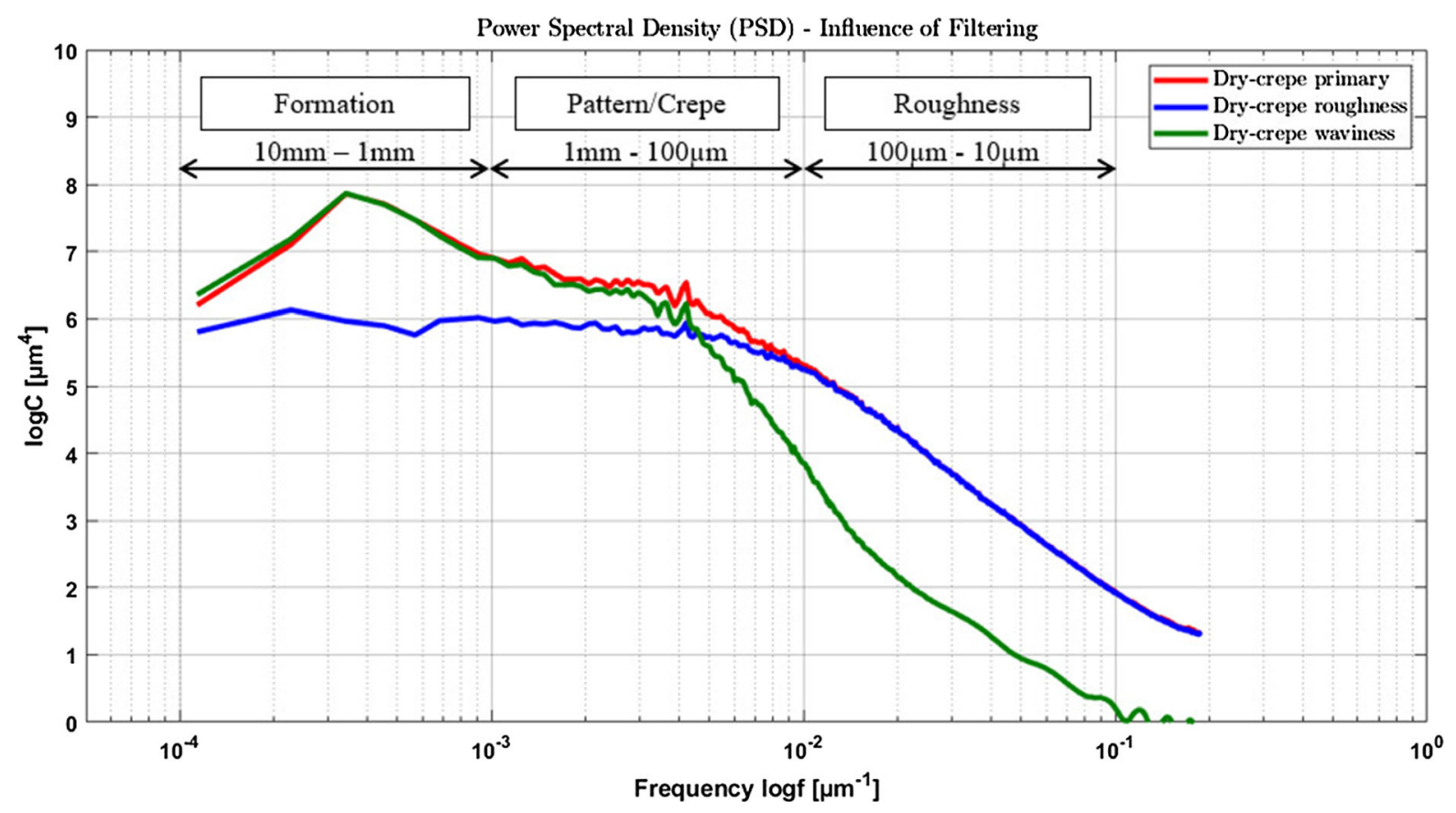

Fig. 5 The spatial averaged PSD curves for the dry-creped tissue surface, which was acquired at a magnification of $10 \mathrm{x}$, are shown separately for the primary and filtered waviness/ roughness profile. A rough classification of the frequency spectra was done. The waviness PSD curve (green) follows and overlaps the primary PSD curve (red) in the lower frequency range, while the roughness PSD curve (blue) overlaps the primary PSD curve in the higher frequency range 
within the whole frequency spectrum and thus shall be used for PSD analysis.

\section{PSD—Effect of magnification}

The level of detail of the obtained topographical data from the IFM depends on the vertical resolution of each magnification (see Table 2), thus the accessible surface information differs. The influence of the used magnification on the 3D surface data is shown exemplary for the textured tissue sample in Figure 6. The benefit of the lowest magnification $(5 x)$ is the possibility to detect large sized structures and patterns while providing a certain periodicity. On the other hand, information regarding small scaled structures, like protruding fibres, is not available. This information can be provided at the highest magnification (20x), where small structures at fibre scale can be observed. Due to the optical measurement method protruding fibres are visible as long, steep flanked regions on the surface. Protruding fibres and also the contours of the fibre network mainly affect the roughness region of the PSD. An extended and valid overview of the frequency spectra is possible when all magnifications are considered. Surface roughness is of course represented more accurately at highest magnification while the medium and lowest magnifications are more precise at lower frequencies. For detailed analysis on surface roughness a magnification of $20 x$ and for structural properties a magnification of $5 \mathrm{x}$ should be used.

\section{PSD evaluation-Comparison of tissue grades}

Considering all discussed aspects of PSD and its application, the three used tissue grades are characterized regarding their surface properties. The corresponding primary PSD curves for roughness evaluation (20x) are shown in Figure 7 as spatially averaged PSD and for structural evaluation $(5 x)$ in Figure 8 as separate PSD curves for MD and CD.
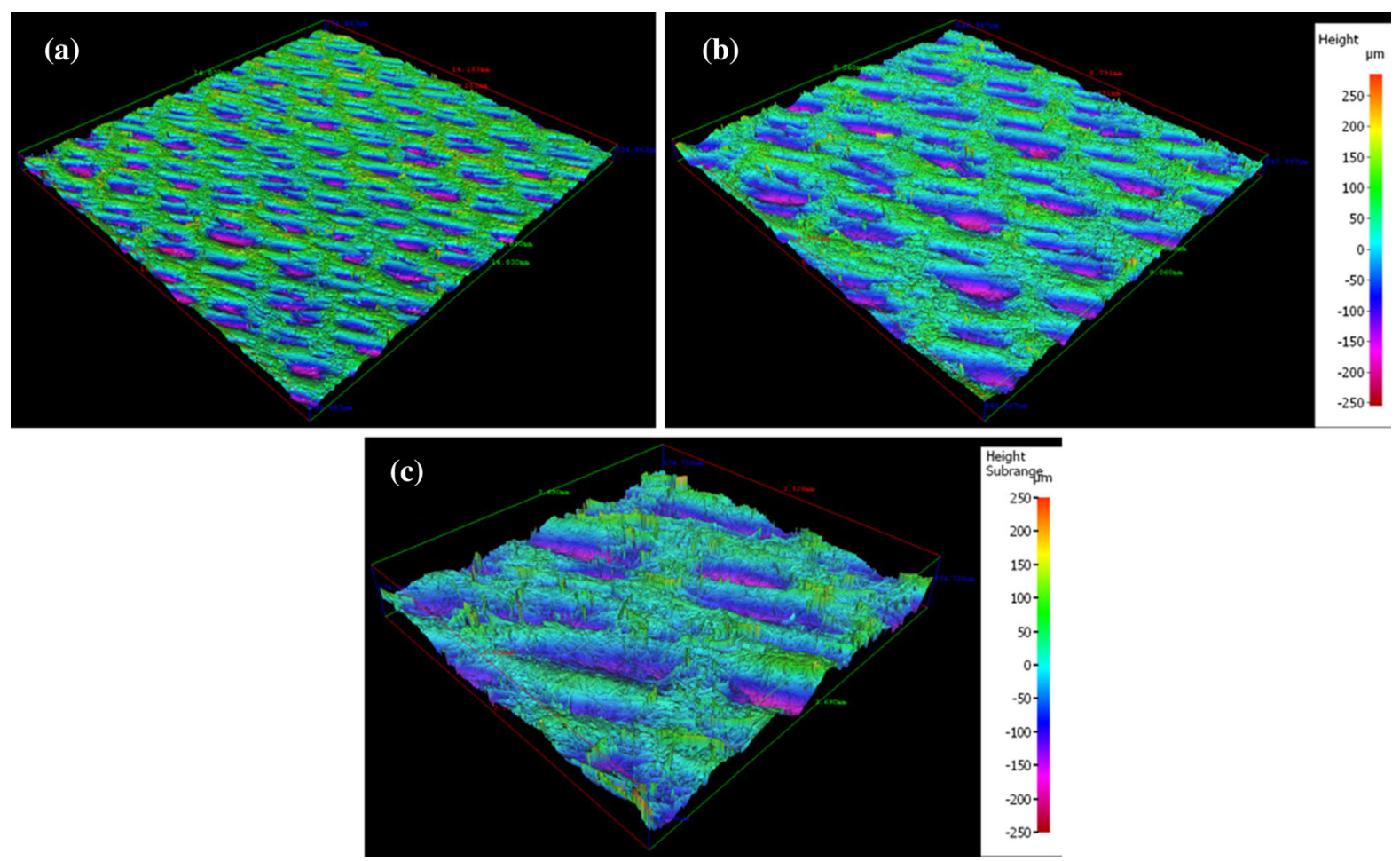

Fig. 6 Surface of textured tissue observed with three magnifications. a Tissue surface at lowest magnification $(5 \times)$ with a lateral size of $14 \times 14 \mathrm{~mm}$, which is used for structural and large scale analysis. b Medium magnification $(10 \times)$ with a lateral size of $8 \times 8 \mathrm{~mm}$ and is used for structural and roughness evaluation. c Highest magnification $(20 \times)$ with a lateral size of $3.5 \times 3.5 \mathrm{~mm}$ and is used for roughness evaluation 
The surface roughness is examined in the frequency range from $8 * 10^{-3}$ to $2 * 10^{-1} \mu \mathrm{m}^{-1}$ at the highest magnification (20x) for each tissue grade. Comparing

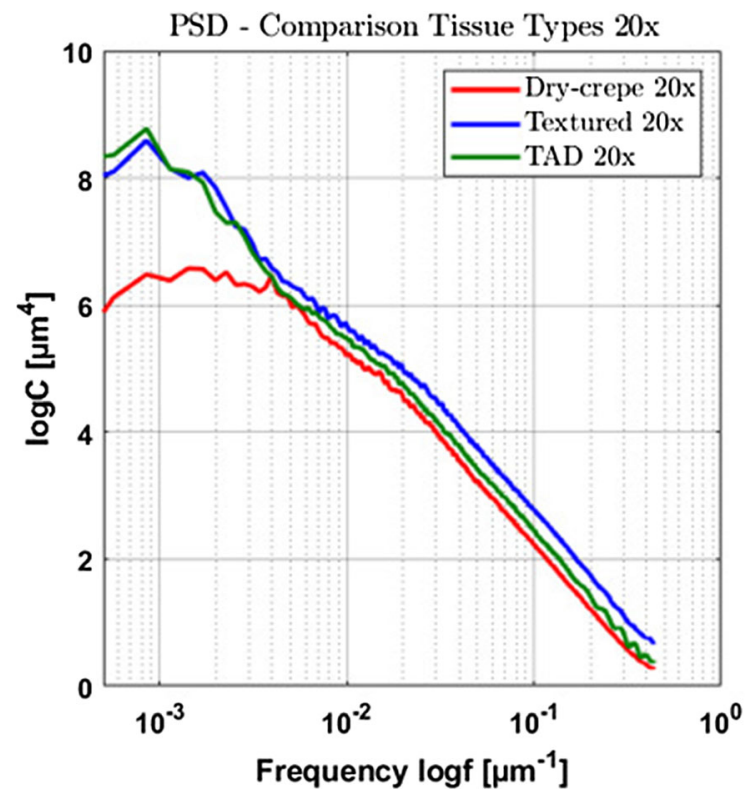

Fig. 7 Surface roughness analysis using spatial averaged PSD curves for the primary dataset of the three tissue grades at highest magnification $(20 \times)$ the roughness PSD curves it is obvious that the textured tissue (blue) shows the highest values, hence the measured surface roughness is higher compared to the dry-creped (red) and TAD tissue (green). TAD shows slightly higher values for surface roughness than the dry-creped tissue. These differences may directly be related to the production process. The drycreping step breaks the mechanical integrity of the network to some extent and therefore leads to fibres randomly protruding from the surface to create surface softness in standard dry creped products. TAD production excludes any mechanical compression and dewatering of the tissue web, which generates a looser network structure with a high roughness. Due to the structured surface of the TAD tissue, the contact area and hence the adhesion to the Yankee cylinder is reduced. The low adhesion between the Yankee and the tissue decreases the influence of the dry-creping step on the tissue surface and less fibres are protruding the network compared to other process configurations. In contrast to $\mathrm{TAD}$, the textured tissue production process includes some mechanical dewatering, a wetcreping step and a dry-creping step, thus it combines the advantages of the TAD and dry-crepe technology regarding the surface structure (looser structure

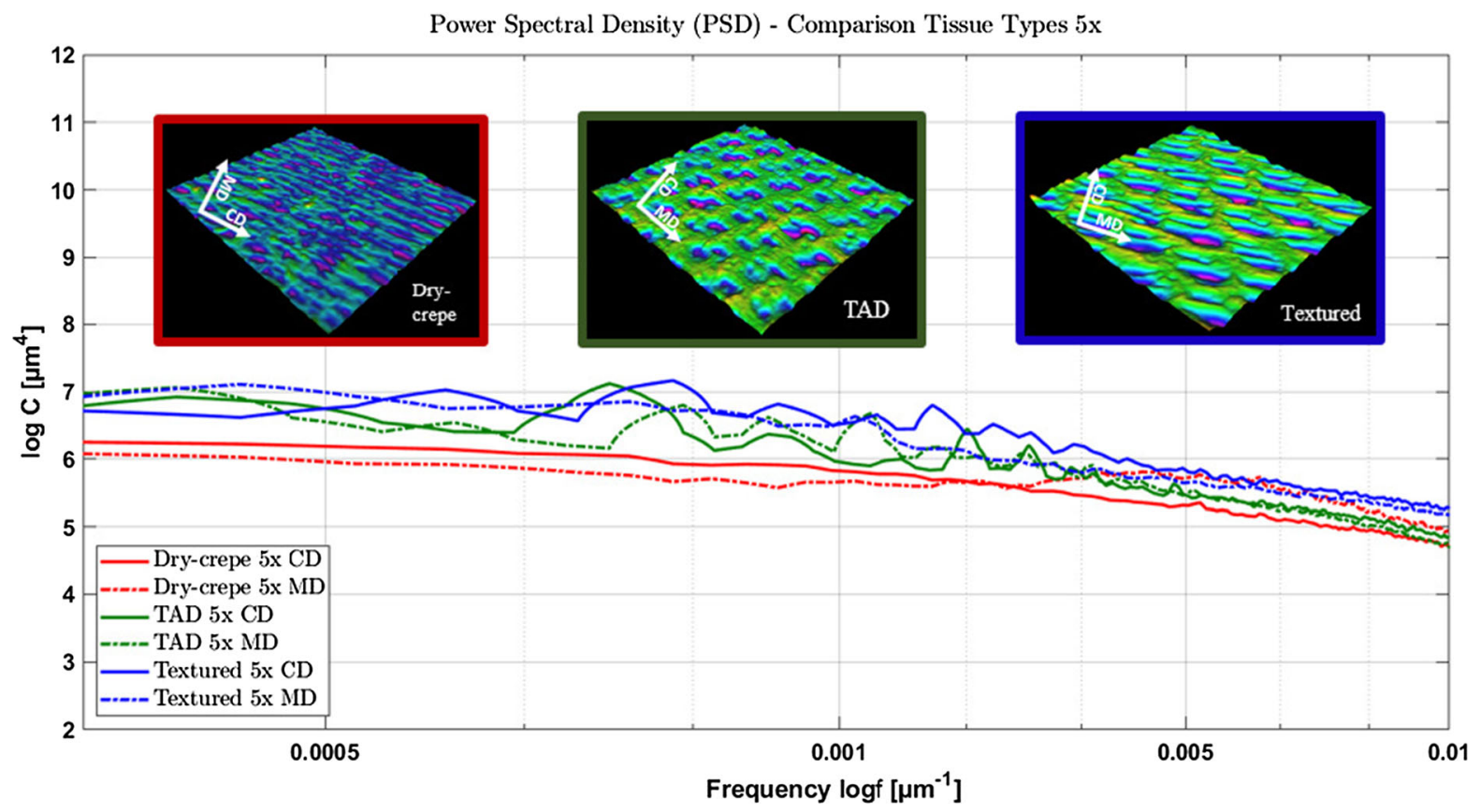

Fig. 8 Separate primary PSD curves for MD and CD of the dry-creped (red), textured (blue) and TAD (green) tissue at a magnification of $5 x$. The corresponding surface topography is shown in a height map, framed with the color of the specific PSD curve 
compared to standard dry crepe + protruding fibres due to extensive creping). In our case the increased amount of randomly protruding fibres are probably the reason for the textured tissues higher surface roughness compared to TAD.

For structural evaluation the PSD curves for the lowest magnification are illustrated in Fig. 8 separately for MD (continuous line) and $\mathrm{CD}$ (dash-dotted line). Additionally the filtered waviness height images of the three tissue grades are shown to provide context. Surface structure due to crepe and fabric patterns are visible in the range from $5 * 10^{-4}$ to $8 * 10^{-3} \mu \mathrm{m}^{-1}$. Differences between the MD and CD PSD curve of the dry-creped tissue are evident. The MD PSD curve shows a higher level as the CD PSD curve in the frequency range around $5 * 10^{-3} \mu \mathrm{m}^{-1}$ which is directly correlated to the periodical MD dry crepe structure. Compared to textured and TAD the drycrepe PSD curves show a certain maximum horizontal level in the range from $1 * 10^{-3}$ to $4 * 10^{-3} \mu \mathrm{m}^{-1}$. The dry-creped structure is mainly affected by the crepe height and regularity (e.g. crepes $/ \mathrm{cm}$ ). The crepe height is low compared to the height of the patterns from the textured and TAD samples, which is also evident in the caliper values in Table 1. Differences in the surface structure between the TAD and textured sample can be detected via the amount and intensity of the peaks in Fig. 8. The textured tissue PSD curves are located above the curves of the TAD on average, which indicates a generally higher height deviance from the valley to the top of the patterns. In addition it shows several small peaks, which are related to various regular patterns in $\mathrm{MD}$ and $\mathrm{CD}$ with different heights and structural sizes. Especially in textured CD several peaks appear over a broad frequency range, which depend mainly on the used fabric. The PSD curves of the TAD sample show intense peaks, which appear at a broad frequency range in $\mathrm{CD}$, while the peaks in MD are shifted to a lower frequency. These differences can be attributed to the molding process during the dewatering and the used fabric on the TAD tissue machine. The analysis and comparison of topography based PSD allows an enhanced view on the characteristics of tissue surfaces and provides a powerful tool for further optimization of tissue surface structures.

Structures for the topographical variance analysis can be obtained only at the lowest magnification $(5 \mathrm{x})$ due to the necessary lateral size. The variation in local mass is crucial in tissue production as it affects the creping and moulding process and thus the surface topography (see Raunio et al. (2012)). The effect of large scaled structures on dry-creped tissue will be discussed separately.

\section{Surface roughness evaluation with $\mathrm{Sdr}$}

Compared to the roughness evaluation with the PSD curve, the developed interfacial area ratio (Sdr) expresses the surface roughness as a figure in percent based on a different principle of surface roughness characterization (see Fig. 3). As it is common in surface analysis to describe and compare surface roughness with a numerical parameter, the Sdr is very suitable for tissue paper. In Table 3 the mean Sdr values for the magnification of 20x are listed. The $\mathrm{Sdr}$ values were evaluated for the primary $(\mathrm{P})$, roughness $(\mathrm{R})$ and waviness $(\mathrm{W})$ profile, which are separated by a Gaussian filter (see Fig. 2). For each tissue grade three arbitrary areas were observed and the results were averaged. The corresponding standard deviations (SD) for each measurement are also shown in Table 3 and indicate a high reproducibility. Advantageously, the $\mathrm{Sdr}$ is nearly independent from textures and patterns on the surface (low Sdr (W) values), thus allowing the comparison of different tissue grades regarding their surface roughness. In contrary to Sdr, height based values like $\mathrm{Sa}$ or $\mathrm{Sq}$ have their limits in comparing tissue with deviations in caliper and structural patterns and should be avoided for characterization of such complex surfaces. Since structures have only a minor influence on Sdr analysis the difference between $\mathrm{Sdr}$

Table 3 Overview of the mean developed interfacial surface areal ratio (Sdr), separately for the primary, roughness and waviness dataset at a magnification of $20 \mathrm{x}$ and the corresponding standard deviations (SD)

Magnification 20x

\begin{tabular}{lrll}
\hline Sdr (P) [\%] & 461.45 & 1098.99 & 517.24 \\
SD (P) [\%] & 22.43 & 5.64 & 8 \\
Sdr (R) [\%] & 453.95 & 1085.24 & 506.72 \\
SD (R) [\%] & 22.04 & 5.66 & 8.66 \\
$\operatorname{Sdr}(W)[\%]$ & 8.34 & 18.82 & 12.76 \\
SD (W) [\%] & 0.44 & 0.69 & 2 \\
\hline
\end{tabular}



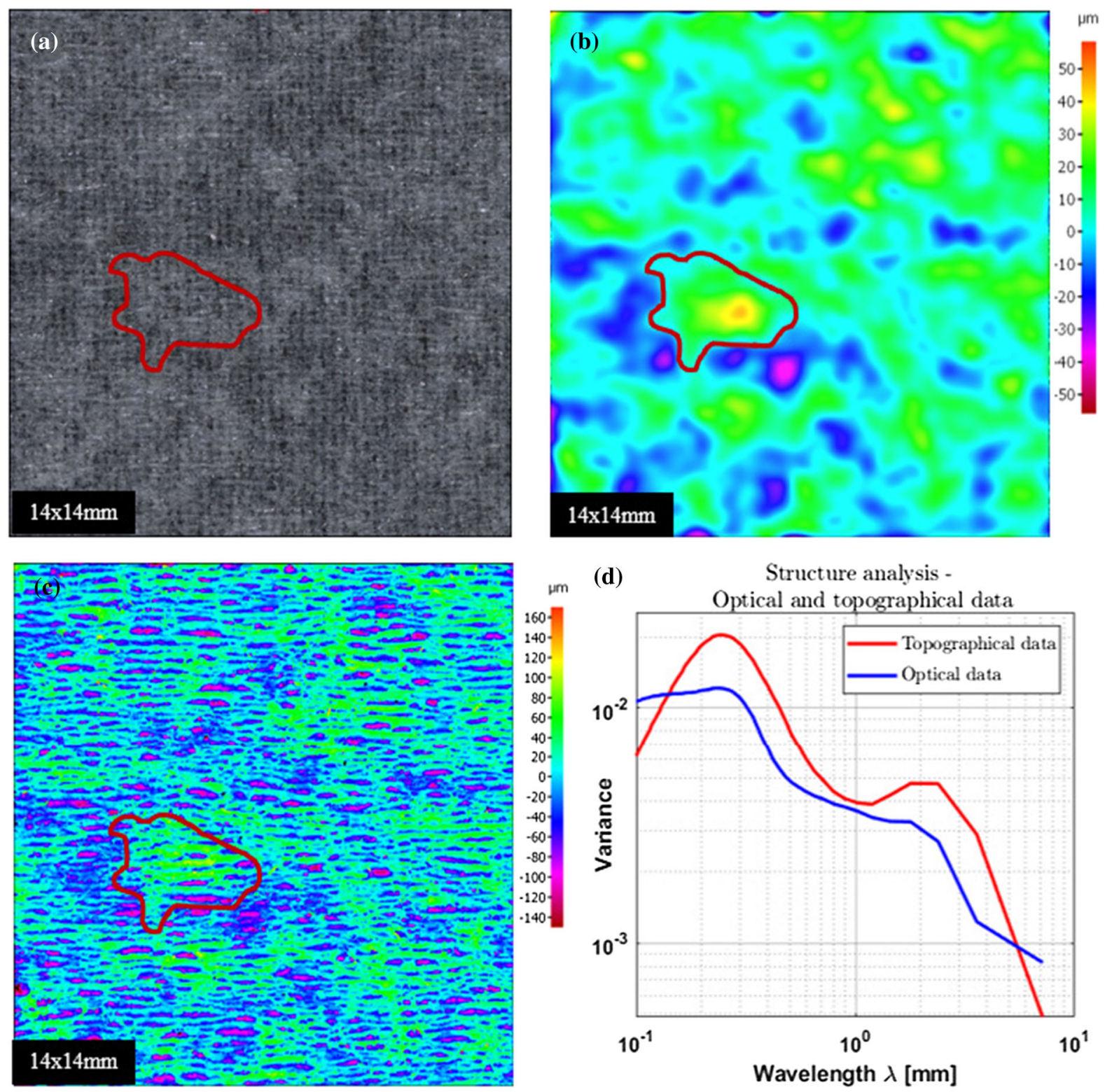

Fig. 9 a Greyscale image of the obtained tissue surface at a magnification of 5x. b Corresponding twice filtered topographical height map without the crepe structure. $\mathbf{c}$ Height map of the primary data. d FFT based structure analysis of the optical and topographical data

(P) representing the unfiltered topography and $\mathrm{Sdr}$ (R) representing solely the roughness profile is hardly significant. This shows that the interfacial area ratio (Sdr) does allow roughness measurement on tissue surfaces without pretreatment of the primary profile. Comparing the samples the textured tissue shows by far the highest Sdr, followed by the TAD and the drycreped tissue which is in accordance with the results from PSD analysis (see Fig. 7).
Optical and topographical variance analysis

The IFM provides a large field of view at lowest magnification $(5 \mathrm{x})$. This allows in addition to the described analysis of surface structures and roughness an optical and also a topographical analysis of largescaled structures. Large structures in topographical variance analysis are identified by repetitive use of the Gaussian filter on the waviness dataset of the lowest 
magnification $(5 \mathrm{x})$ to eliminate smaller structures (e.g. crepe, fabric). In Fig. 9 the optical greyscale image (a), the corresponding filtered topographical height map (without the crepe-structure) (b) and the primary surface height map (c) for the dry-creped tissue are shown. In the primary height map (c) the crepe is visible as the dominant structure, with the frequency of the crepe varying throughout certain regions (such as in the region marked in red). It does seem that the crepe frequency is somewhat related to the underlying structure. The kind of relation would have to be clarified in future work. The inhomogeneity in the underlying structure of the paper is accessible with focus variation as is shown in the height map (b) and could have different reasons like e.g. formation effects in the wet end of the tissue machine or in-plane deviations due to the drying process. In the optical greyscale image (a) similar large scale variations are visible due to differences in light reflectance. This similarity in structures of the topographical and optical data is shown in the structure analysis in (d). In this analysis the primary topography image (c) was converted to a greyscale image and both, the optical and topographical image, were subsequently normalized and subjected to FFT based structure analysis. Beside the peaks at a wavelength of $2.5 * 10^{-1} \mathrm{~mm}$ (which represent the crepe), there is an increase in variance for both datasets at a wavelength of $2 \mathrm{~mm}$. This indicates a coherence between the optical grey scale image and topographical data that may be related to e.g. formation. Still, further work regarding topographical and optical large scale structure analysis and the reasosn for its coherence is necessary.

\section{Conclusion and outlook}

With the used infinite focus technology enhanced analysis of complex tissue surfaces is possible. The applicability can be extended to several fibre based materials with similar optical properties as tissue paper such as e.g. nonwovens. The optical method in combination with the developed procedure is fast, flexible, error robust and has a high capability compared to other surface measurement devices. The measured 3D datasets, consisting of a dry-creped, a textured and a TAD tissue sample are pre-treated to minimize optical errors for further assessment. The areal surface analysis considers the whole measured area and provides special parameters for surface evaluation. The power spectral density (PSD) is an appropriate tool to evaluate the whole frequency spectra of surface structures like crepe, fabric-based patterns and roughness simultaneously. The use of different magnifications extends the valid frequency range of the PSD curve, thus wavelengths from $10 \mu \mathrm{m}$ up to $10 \mathrm{~mm}$ can be evaluated. Dominant structures are apparent as peaks and can be related to a certain wavelength. Changes in process conditions can be determined by a shift of the intensity and frequency of these peaks. Additionally to PSD evaluation surface roughness can be described with the developed surface area ratio (Sdr). PSD and Sdr are based on different concepts, although they show similar results regarding surface roughness and should be used primarily for complex surfaces like tissue. Both parameters can be considered as a potential measure to describe handfeel related properties like tissue surface softness in future work. Due to the high field of view of the infinite focus device and the possibility to obtain optical images, the method is also suitable to evaluate the optical variations in combination to the topographical information. Summming up, this work shows the potential of optical based tissue surface analysis by means of focus variation. Such a detailed surface analysis is crucial for a better understanding of tissue surface and human skin interactions.

Acknowledgments We acknowledge the support by our industrial partner Andritz AG and the Austrian Research Promotion Agency (FFG). We also want to mention BrukerAlicona for their software support.

Author Contributions All authors contributed to the study conception and design. Material preparation, data collection and analysis were performed by Reitbauer Jürgen. The first draft of the manuscript was written by Reitbauer Jürgen and all authors commented on previous versions of the manuscript. All authors read and approved the final manuscript.

Funding Open access funding provided by Graz University of Technology. This study was funded by the Austrian Research Promotion Agency (FFG)

Availability of data and materials The datasets generated and/or analysed during the current study are available from the corresponding author on reasonable request.

Code availability Commercial software was used. 


\section{Declarations}

Conflict Interest The authors have no relevant financial or non-financial interests to disclose.

Ethics approval This chapter does not contain any studies with human participants or animals performed by any of the authors.

Open Access This article is licensed under a Creative Commons Attribution 4.0 International License, which permits use, sharing, adaptation, distribution and reproduction in any medium or format, as long as you give appropriate credit to the original author(s) and the source, provide a link to the Creative Commons licence, and indicate if changes were made. The images or other third party material in this article are included in the article's Creative Commons licence, unless indicated otherwise in a credit line to the material. If material is not included in the article's Creative Commons licence and your intended use is not permitted by statutory regulation or exceeds the permitted use, you will need to obtain permission directly from the copyright holder. To view a copy of this licence, visit http://creativecommons.org/licenses/by/4.0/.

\section{References}

Blateyron F (2006) New 3D parameters and filtration techniques for surface metrology. In: JSPE, pp 1-7

Bruker-Alicona (2019) MeasureSuite Manual 5.3.5

Danzl R, Helmli F, Scherer S (2011) Focus variation - a robust technology for high resolution optical 3D surface metrology. J Mech Eng 57(3):245-256. https://doi.org/10.5545/ sv-jme.2010.175

de Assis T, Reisinger LW, Pal L, Pawlak J, Jameel H, Gonzalez RW (2018) Understanding the effect of machine technology and cellulosic fibers on tissue properties - a review. BioResources. https://doi.org/10.15376/biores.13.2. deassis

de Assis T, Pawlak J, Pal L, Jameel H, Venditti R, Reisinger LW, Kavalew D, Gonzalez RW (2019) Comparison of wood and non-wood market pulps for tissue paper application. BioResources 14(3):6781-6810. https://doi.org/10. 15376/biores.14.3.6781-6810

Furman G, De Roever E, Frette G, Gomez S (2010) Analysis of the surface softness of tissue paper using confocal laser scanning microscopy. Celulosa Y Papel 26(3):30-38

Gigac J (2019) Prediction of water-absorption capacity and surface softness of tissue paper products using photoclinometry. O Papel 80(08):91-97

Hollmark H (1983) Evaluation of tissue paper softness. Tappi J 66(2):97-99

Hollmark H, Ampulski RS (2004) Measurement of tissue paper softness: A literature review. Nord Pulp Paper Res J 19(3):345-353. https://doi.org/10.3183/npprj-2004-19-03p345-353

Ismail MY, Patanen M, Kauppinen S, Kosonen H, Ristolainen M, Hall SA, Liimatainen H (2020) Surface analysis of tissue paper using laser scanning confocal microscopy and micro-computed topography. Cellulose. https://doi.org/10. 1007/s10570-020-03399-w

Jacobs TD, Junge T, Pastewka L (2017) Quantitative characterization of surface topography using spectral analysis. Surf Topogr Metrol Prop. https://doi.org/10.1088/2051672X/aa51f8. http://arxiv.org/abs/1607.03040

Kawabata S (1980) The Standardization and Analysis of Hand Evaluation, 2nd edn. The Textile Machinery Society of Japan, Osaka

Ko YC, Park JY, Lee JH, Kim HJ (2017) Principles of developing a softness evaluation technology for hygiene paper. Palpu Chongi Gisul/J Korea TAPPI 49(4):184-193. https:// doi.org/10.7584/JKTAPPI.2017.08.49.4.184

Ko YC, Melani L, Park NY, Kim HJ (2019) Surface characterization of paper and paperboard using a stylus contact method. Nord Pulp Paper Res J. https://doi.org/10.1515/ npprj-2019-0005

Leach R (2011) Optical Measurement of Surface Toporgraphy. 1st edn. Springer Berlin, Heidelberg. https://doi.org/10. 1007/978-3-642-12012-1. http://arxiv.org/abs/1011.1669

Leach R (2013) Characterisation of areal surface texture. https:// doi.org/10.1007/978-3-642-36458-7

Lechthaler M, Bauer W (2006) Rauigkeit und Topografie - Ein Vergleich unterschiedlicher Messverfahren. Wochenblatt fuer Papierfabrikation 134(21):1227-1234

Lee SH, Kim HJ, Ko YC, Lee JH, Park JY, Moon BG, Park JM (2017) Characterization of Surface Properties of Hygiene Paper by Fractal Dimension Analysis Technique. J Korea TAPPI 49(6):90-101. https://doi.org/10.7584/JKTAPPI. 2017.12.49.6.90

Mettänen M, Hirn U (2015) A comparison of five optical surface topography measurement methods. Tappi J 14(1):27-38

Park NY, Melani L, Kim HJ, Lee JJ, Woo KS (2019) Determination of the tensile modulus of facial tissue. Palpu Chongi Gisul/J Korea TAPPI 51(5):105-112. https://doi. org/10.7584/JKTAPPI.2019.10.51.5.105

Pawlak JJ, Elhammoumi A (2011) Image Analysis Technique for the Characterization of Tissue Softness. In: Progress in Paper Physics Seminar, Graz, pp 231-238. https://doi.org/ 10.3217/978-3-85125-163

Raunio JP, Ritala R, Mäkinen M (2012) Variability of crepe frequency in tissue paper; relationship to basis weight. Paper Conf Trade Show 2012, PaperCon 2012 2:910-920

Raunio JP, Löyttyniemi T, Ritala R (2018) Online quality evaluation of tissue paper structure on new generation tissue machines. Nord Pulp Paper Res J 33(1):133-141. https://doi.org/10.1515/npprj-2018-3004

Rosen BG, Fall A, Rosen S, Farbrot A, Bergström P (2014) Topographic modelling of haptic properties of tissue products. Conf Ser, J Phys. https://doi.org/10.1088/17426596/483/1/012010

Rust JP, Keadle TL, Allen DB, Shalev I, Barker RL (1994) Tissue softness evaluation by mechanical stylus scanning. Text Res J 64(3):163-168. https://doi.org/10.1177/ 004051759406400306

Vernhes P, Bloch JF, Mercier C, Blayo A, Pineaux B (2008) Statistical analysis of paper surface microstructure: A multi-scale approach. Appl Surf Sci 254(22):7431-7437. https://doi.org/10.1016/j.apsusc.2008.06.023

Wang Y, De Assis T, Zambrano F, Pal L, Venditti R, Dasmohapatra S, Pawlak J, Gonzalez R (2019) Relationship 
between human perception of softness and instrument measurements. BioResources 14(1):780-795. https://doi. org/10.15376/biores.14.1.780-795

Wanske M, Qroßmann H, Scherer S (2008) Messtechnische Bewertung der Glätte von Tissue-produkten mit dem Optischen Messsystem InfiniteFocus. Wochenblatt fuer Papierfabrikation 136(9):473-477
Publisher's Note Springer Nature remains neutral with regard to jurisdictional claims in published maps and institutional affiliations. 